\title{
Formación en las aulas normalistas
}

\section{Formation on the classrooms of teaching college students}

\author{
Silvia Chacón Flores ${ }^{1}$
}

\section{Resumen}

El presente trabajo muestra avances de la investigación educativa cuya temática es la formación profesional docente, misma que está en proceso, actualmente se encuentra con avances del 85 por ciento; además representa una de las categorías que conforman una investigación mayor titulada "Formación profesional" la cual se desarrolla al interior de un equipo de investigación que está en proceso de registro. En este trabajo se pretende mostrar la identidad profesional dentro de su formación inicial como docentes de la educación básica, en las licenciaturas de Educación Primaria y Educación preescolar; todo ello a partir del enfoque cualitativo para describir el impacto de la formación normalista en los futuros docentes.

\section{Palabras clave}

Formación, identidad profesional.

\begin{abstract}
The present work gives to show the research advances of education whose subject is teacher professional training which is in process, is currently with advances of 85 percent; It also represents one of the categories that make up a major research entitled "Vocational Training" which is developed within a research team that is in the process of registration. This paper aims to show the professional identity within their initial training as teachers of basic education, in the Bachelor of Primary Education and Early Childhood Education; all this based on the qualitative approach to describe the impact of normal education on future teachers.
\end{abstract}

${ }^{1}$ Silvia Chacón Flores. Docente de la Institución Benemérita y Centenaria Escuela Normal del Estado de Chihuahua Profr. Luis Urías Belderráin, México. Es maestra en educación campo práctica docente y candidata a doctora. Correo electrónico: silviachflores10@hotmail.com

ID: http://orcid.org/0000-0001-5523-5542 
RECIE. Revista Electrónica Científica de Investigación Educativa Vol. 4, núm. 2, enero-diciembre 2019, pp. 1119-1127.

\section{Keywords}

Formation, profesional identity.

\section{Introducción}

La sociedad del siglo XXI presenta cambios vertiginosos en todos los aspectos, posee una realidad compleja en sí misma, la sobreproducción de información, los logros humanos en las ciencias, el avance de la tecnología y la ideología del mundo globalizante contrasta de manera dramática con la exacerbación de la pobreza, la inequidad y la desigualdad al acceso del conocimiento y de la movilidad social.

En las escuelas normales el deber ser, está implícito en la currícula y en la exigencia de cubrir con el plan y programa de estudio, se ha descuidado la parte emotiva de la profesión, la deshumanización se ve plasmada por la respuesta que se da a la concepción empresarial de la labor educativa.

La presente investigación, como ya se dijo en el resumen, aún no está concluida, posee un $85 \%$ aproximadamente. Su temática obedece a la formación profesional docente que se desarrolla al interior de las aulas normalistas de la IByCENECH asociada a la identidad, en los segundos y octavos semestres de las Licenciaturas de Educación Preescolar y Primaria. El enfoque para abordar el tema es de corte cualitativo, toda vez que pretende dar voz a los sujetos de investigación para que narren sus experiencias. El propósito que aquí se persigue es conocer y describir los procesos de formación que siguen los alumnos que ingresan y egresan a la institución relacionada con la identidad profesional y los cambios que se dan a lo largo de su trayecto formativo como docente.

\section{Planteamiento del problema}

En México se vive un intento por aplicar una reforma educativa, que por su contenido fuertemente labora, ha generado el rechazo generalizado del profesorado de la educación básica; a esta reforma la acompaña el desprestigio social del maestro mexicano y un panorama poco propicio para la visualización de la docencia como una profesión atractiva. En la IByCENECH se ha reflejado esta polémica, en la matrícula. En años anteriores la demanda era muy alta, más de la mitad de los aspirantes quedaban fuera; situación que actualmente no se le iguala. Esta institución, con más de 100 años de egresar maestros identificados con su labor y que han aportado una visión de la docencia como carrera de vid, a la sociedad 
Chihuahuense y al país, hoy se ve afectada al tener pocos aspirantes de ingreso.

La formación inicial docente de la educación básica requiere de un análisis profundo, de incursionar en un campo polémico, pues en la actualidad, la educación es un tema a investigar que requiere atención inmediata. Por esta razón es de carácter importante dar a conocer el proceso de formación que se sigue en las licenciaturas y los sujetos de investigación para conocer su recorrido inicial, de igual manera, saber si responde a sus expectativas y necesidades. El problema detectado implica, en caso de nula o poca atención, un alto riesgo institucional, debido a que la razón de su existencia es precisamente atender jóvenes que desean desempeñarse el área de la educación, de tal manera que si continúa con pocos aspirantes a ella se puede generar un problema de atención o disminución de la calidad educativa que por muchos años se ha brindado en esta casa de estudios.

La pregunta general que conduce esta investigación es: ¿Cómo es el proceso de formación que recorren los alumnos y alumnas de las licenciaturas de educación preescolar y primaria de la IByCENECH para formar o consolidar la identidad profesional?

Este último factor, la identidad, debe dar a conocer las causas por las que los futuros profesores ingresan a la normal; puesto que la diversidad de razones al respecto pueden considerarse como una influencia en la construcción de esa identidad. El problema se hace evidente a partir de la puesta en marcha del plan de estudios 2012 y la baja matrícula presentada en los dos últimos ciclos escolares.

Las preguntas particulares son:

- ¿Cuáles son las características de los alumnos de nuevo ingreso y sus expectativas con respecto de la formación docente?

- ¿Cuáles son los momentos por los que atraviesa el maestro en formación para lograr sus competencias propuestas en Plan y Programa de estudio de las licenciaturas de Educación Preescolar y Educación Primaria?

- ¿Qué papel juega la Escuela Normal en la construcción de la Identidad Docente de los estudiantes de la Licenciatura en Educación Preescolar y Educación Primaria?

\section{Objetivo}

Analizar el proceso de formación que recorren los futuros docentes de la IByCENECH y la influencia de la Escuela Normal en la construcción de la 
identidad docente de los estudiantes de las Licenciaturas en Educación Preescolar y Educación Primaria.

En este momento histórico se hace de suma importancia realizar una indagación respecto al tema, debido a que existe una fuerte necesidad de docentes comprometidos con su actividad, ya que los cambios respecto a la concepción del trabajo y profesión docente traen como consecuencia una fuerte presión social sobre el rol del maestro, obliga y compromete a responder la identidad y formación de los futuros maestros, tanto en la IByCENECH como del resto de las escuelas normales del Estado y del país.

Por otro lado, la investigación debe encontrar aquellos factores que dificultan o favorecen la identidad con la docencia, debe aportar también información con miras a mejorar por parte de los formadores de formadores, de responder a las expectativas que los mismos maestros en formación manifiestan.

Los mayores beneficiados de esta investigación son los mismos alumnos de la IByCENECH pues son ellos quienes enfrentan las razones por las que llegaron a esta casa de estudios y por las que concluyen su formación inicial docente. De esta manera el equipo de investigación hará aportaciones positivas a la formación docente de la institución donde laboran y de donde egresaron, pues en ella no existen datos que den muestra que se han realizado investigaciones al respecto.

\section{Fundamentación teórica}

La formación en términos generales es motivo de debate, la formación profesional docente lo es más.

Gilles Ferry (Gilles, 1997 p. 111) afirma que la formación profesional presupone conocimientos, habilidades, cierta representación del trabajo a realizar, la concepción del rol, etc. Explica que la formación es un desarrollo de cada individuo, que va orientando según los objetivos que busca de acuerdo a su posición. Una formación no se recibe, se construye.

El Consejo Universitario de las Ciencias Exactas y Naturales (2011, p. 30) concibe a la formación como un conjunto de acciones diseñadas y ejecutas por las autoridades educativas y las instituciones de educación superior para proporcionar al personal bases teóricas y prácticas de la pedagogía.

Bernard Honoré (Honoré, 1998 p. 56) afirma que la formación, hoy en día se entiende o se conceptualiza principalmente como una serie de actividades. Analiza el término a partir de su asociación a otros, como formación personal, profesional, política, etc. Considera, además, que cada 
uno tiene su propio concepto y que generalmente se toma como "derivado de formar" y aplicado al ser humano significaría "desarrollar las capacidades naturales, hacer capaz de alguna función determinada, o generalmente realizar su finalidad de hombre". Siempre se trata de formar algo, como una actividad por la cual, se busca, con el otro, las condiciones para un saber exterior, luego interiorizado, bajo una nueva forma.

Otra noción que se debe tener presente en esta investigación es la formación inicial en términos generales y asociada a la docencia. La cual es un proceso sistemático y organizado, dicen Cornejo (2012) que posibilita el desarrollo de competencias propias del ejercicio profesional en los diferentes niveles del sistema educacional. En este proceso, no sólo se incluyen contenidos específicos, sino que, además ciertas habilidades o estrategias que tienen que ver con ciertas cualidades personales y sociales que este futuro profesional debe poseer.

El concepto competencia remite a la idea del aprendizaje significativo. El término en sí, tiene múltiple acepciones, según la UNESCO es la capacidad expresada mediante los conocimientos, las habilidades y las actitudes, que se requiere para ejecutar una tarea de manera inteligente, en un entorno real o en otro contexto. (UNESCO, 2009, s/p).

Las competencias profesionales a desarrollar, propuestas en el Plan y Programas de estudio de la Licenciatura en Educación Preescolar y en Educación Primaria permitirán a los egresados atender situaciones y resolver problemas en los diferentes contextos escolares en los que deberá incursionar (SEP, 2012).

Otra categoría que se debe conceptualizar es la identidad profesional. La cual se entiende como una construcción dinámica, influida por factores individuales y sociales, que implican una parte racional (una identificación de conocimientos específicos de la profesión) y otra emocional (los sentires respecto al ser docente) lo cual lleva a un individuo a identificarse con lo que hace, con una actitud comprometida y responsable (Vaillant, 2008) la temática de la identidad docente refiere a cómo los docentes viven subjetivamente su trabajo y a cuáles son los factores de satisfacción e insatisfacción. También guarda relación con la diversidad de sus identidades profesionales con la percepción del oficio por los docentes mismos y por la sociedad.

\section{Metodología}

"El método es un discurso, un ensayo prolongado de un camino que se piensa. Es un viaje, un desafío, una travesía, una estrategia que se ensaya para llegar a un final pensado, imaginado y al mismo tiempo insólito, 
imprevisto y errante. No es el discurrir de un pensamiento seguro de sí mismo, es una búsqueda que inventa y se reconstruye continuamente" (Morin, 2009).

Es evidente que toda investigación se realiza desde una plataforma o matriz conceptual, al cual muchos investigadores y teóricos los llaman paradigma, el cual define las características del objeto de investigación, los problemas que plantear y resolver, la propia esencia de los productos, las estrategias, técnicas e instrumentos que se consideran más adecuados.

La presente investigación se trata de un estudio que pretende, a través de un enfoque cualitativo, explorar los procesos de construcción o consolidación de la identidad docente que vivencia los alumnos de las Licenciaturas en Educación Preescolar y Primaria de la IByCENECH. Se elige la investigación cualitativa ya que, serán los participantes quien es relaten sus vivencias y expectativas en relación a su formación profesional docente, así como aquellos aspectos con los que se identifica con la profesión.

\section{Sujetos de investigación y tipo de muestreo}

Los sujetos a investigar son los alumnos que en el presente ciclo escolar cursan el segundo y octavo semestre de las licenciaturas en Educación Preescolar y Educación Primaria. La muestra se eligió por conveniencia de quien realiza la investigación. El tipo de muestreo se realizó con base al tipo No probabilísticas; ya que según Hernández Sampieri (Hernández, 2010, p. 135) este tipo de muestras se da cuando la elección de los elementos no depende de la probabilidad, sino de causas relacionadas con el investigador o del que hace la muestra. Los grupos a los cuales se les apli8caron los instrumentos de la investigación, fueron aquellos a los que se tuvo acceso y disposición.

\section{Instrumentos de investigación}

Los instrumentos de investigación después de un proceso de construcción se pilotearon en grupos distintos seleccionados como sujetos de investigación. Consisten en una encuesta para cada alumno, tanto de segundo como de octavo semestre. La encuesta según Audirac (Audirac, 2009 , p. 60) y otros "es un instrumento que permite recabar información general y puntos de vista de grupos de personas", en este caso, de sujetos de investigación. A través de este instrumento se va a recolectar actitudes, opiniones y aquellos datos de la población a investigar, esto permite abordar y analizar las categorías de análisis formuladas a lo largo de la investigación. 
Otra técnica de investigación a utilizar es el grupo focal. Al ser éste un espacio de opinión para captar con mayor fidelidad al sentir, el pensar y el vivir de los sujetos de investigación, induciendo a las auto-explicaciones para obtener datos cualitativos.

Tanto las respuestas de las encuestas como los resultados de los grupos focales, se analizarán con la herramienta tecnológica Atlas ti para ayudar a analizar los datos cualitativos, las frecuencias, establecer vínculos entre categorías. Luego de obtener los resultados concentrados, se procederá al análisis y triangulación correspondientes.

\section{Resultados y conclusiones}

Rodríguez, Gil y García, (1996, p 46) comentan que:

Los resultados de un estudio avanzarán en la explicación, comprensión y conocimiento de la realidad educativa y contribuirán a la teorización o intervención sobre la misma. La investigación de corte cualitativo se preocupa especialmente por el significado que los participantes atribuyen a sus prácticas y a las situaciones educativas en las que se desarrollan. Los resultados o conclusiones de esta investigación englobarán, por tanto, toda una serie de decisiones del investigador sobre el significado de las cosas. (s/p).

Los avances realizados son la fundamentación teórica y metodológica, la construcción de los instrumentos, el análisis de la viabilidad y la sensibilización de los participantes de la investigación, las autoridades institucionales y las demás personas que laboran en la institución y que de alguna manera se ven involucrados en este proceso investigativo. Se aplicaron encuestas a los alumnos de 2 grupos de primer semestre de la Licenciatura en Educación primaria y a un grupo de educación Preescolar. Las categorías de análisis surgieron a partir de las incidencias encontradas que revelan significados potenciales, son conceptos derivados de los datos, que representan fenómenos de la realidad, ordenadores epistemológicos en los cuales, los sujetos de investigación, revelan sus vivencias y experiencias, su contexto.

En dichas encuestas, los maestros en formación de ambas licenciaturas manifiestan que la empatía o vocación por la profesión docente se ha obtenido desde su infancia, misma que se ha visto favorecida por la influencia positivas de sus familiares cercanos que se dedican a la docencia, en otros casos la identidad profesional ha sido fomentada o inspirada por algún maestro significativo, alguien que en algún momento de su formación en alguno de los niveles educativos por los que ha transitado, dejó un agrado hacia la profesión. 
En la categoría de la formación en la Normal, señalan que esperan que la Institución formadora de docentes les proporcione herramientas metodológicas y enfoques necesarios para desempeñarse de manera asertiva al momento de estar en el ámbito laboral. Las expectativas con respecto de su desempeño docente, son altas pues desean ser docentes de éxito, innovadores y ser partícipes de la educación de calidad para aquellos alumnos que acudan a sus aulas.

En cuanto a su formación inicial que realizan en la escuela normal esperan obtener las herramientas y los conocimientos necesarios para desempeñarse de buena manera en las escuelas de educación básica, así como realizar prácticas foráneas en la mayoría de los contextos que conforman el Estado. Consideran, además, que las prácticas educativas son una gran fortaleza, pues les permiten ver, analizar $y$ adquirir conocimientos para enfrentar las diversas situaciones y dificultades que se les presentan en la cotidianidad escolar.

Otro de las categorías de análisis en la presente investigación, es la formación que deben poseer los docentes en cuanto a las habilidades y los conocimientos que se deben desarrollar a lo largo de su formación inicial y manifiestan un listado en el que mencionan: "el conocimiento de todas las asignaturas que se abordan en la educación básica”, el conocimiento y uso de las TIC, conocimientos psicológicos con el fin de conocer de mejor manera a los alumnos que atienden; ser responsable, humanista, puntual, congruente con sus valores; tener el gusto por la lectura, sentido común...

En la categoría "preparación académica que debe tener un docente de cualquier lugar del Estado de Chihuahua" responden de manera generalizada que el nivel licenciatura en educación es el punto de partida para ser competentes, además de no conformarse con ese grado como único, están en actualización permanente.

Para los jóvenes encuestados hasta este momento la identidad profesional es lo que debe caracterizar a todos los docentes, los que están en ejercicio y los que están en formación. Afirman, en su mayoría que la identidad profesional se va desarrollando a lo largo de su preparación y que se concepto se resume en atributos, conocimientos, habilidades y amor que se poseen hacia la docencia.

En cuanto a qué requisitos o características se deben tomar en cuenta para el ingreso al normal, además del examen de admisión; los maestros en formación consideran que se deben poseer las siguientes características:

- Vocación 
- Liderazgo

- Amor a la profesión

- Calificaciones

- Habilidades para ser guías del conocimiento

Y sólo en un caso se menciona que es importante tomar en cuenta si se posee una discapacidad.

Las encuestas que se aplicaron y que proporcionan la información aquí vertida, son sólo tres grupos, uno de la licenciatura de educación preescolar y dos de la licenciatura en educación primaria. Por cuestiones de fechas de jornadas de práctica y algunas circunstancias de índole política laboral han dificultado la aplicación del resto de las encuestas, así como la organización de los grupos focales.

Se pretende, en próximas fechas, estar en condiciones de obtener la totalidad de las respuestas a las encuestas y la realización de los grupos focales para proceder al análisis de los datos obtenidos. También se aplicarán las encuestas a los grupos de octavo semestre, analizar los resultados y proceder al análisis de las categorías encontradas. Otro de los propósitos que se pretende lograr, es extender esta investigación a las escuelas normales del Estado, pues al llevar el mismo programa de estudios, se puede apreciar a simple vista que los egresados poseen diferentes características.

\section{Referencias}

Audirac, C. (2009). Sistematización de la Práctica Docente. Trillas.

Bar, G. (1999). Perfil y competencias del docente en el contexto institucional educativo (documento en línea).

Ferry, G. (1990). El trayecto de la formación docente. Paidós educativo.

Hernández, F. y. (2010). Metodología de la investigación. México: Mac GrawHill.

Honoré, B. (1998). Para una teoría de la formación. Madrid, España: NARCEA.

Morin, E. (2009). Introducción al pensamiento complejo. GEDISA.

Morin, E. (2009). Introducción al pensamiento complejo. GEDISA.

Rodríguez, G., Gil, J., y García, E. (1996) Metodología de la investigación

cualitativa. Granada, España: Ediciones. Aljibe

SEP. (2012). Plan de estudios 2012. México: Secretaría de Educación

Pública.

UNESCO. (2009). Conocimiento Complejo. Texto en línea.

Vaillant, D. (2008). La identidad docente. 
RECIE. Revista Electrónica Científica de Investigación Educativa Vol. 4, núm. 2, enero-diciembre 2019, pp. 1119-1127. 